OPEN ACCESS

Edited by:

Merce Pallas,

University of Barcelona, Spain

Reviewed by:

Kamil Kuca,

University of Hradec Králové, Czechia

Patrizia Giannoni,

University of Nimes, France

Sylvie Claeysen,

Institut National de la Santé et de la

Recherche Médicale (INSERM),

France

*Correspondence:

Giuseppe Di Fede

giuseppe.difede@istituto-besta.it

Received: 28 August 2019 Accepted: 01 November 2019

Published: 15 November 2019

Citation:

Catania M, Giaccone G, Salmona M, Tagliavini $F$ and Di Fede $G$

(2019) Dreaming of a New World Where Alzheimer's is a

Treatable Disorder.

Front. Aging Neurosci. 11:317. doi: 10.3389/fnagi.2019.00317

\section{Dreaming of a New World Where Alzheimer's Is a Treatable Disorder}

\author{
Marcella Catania ${ }^{1}$, Giorgio Giaccone ${ }^{1}$, Mario Salmona ${ }^{2}$, Fabrizio Tagliavini ${ }^{1}$ \\ and Giuseppe Di Fede ${ }^{1 *}$
}

\begin{abstract}
'Neurology V-Neuropathology Unit and Scientific Directorate, Fondazione IRCCS Istituto Neurologico Carlo Besta, Milan, Italy, ${ }^{2}$ Department of Molecular Biochemistry and Pharmacology, Istituto di Ricerche Farmacologiche Mario Negri IRCCS, Milan, Italy
\end{abstract}

Alzheimer's disease (AD) is the most common form of dementia. It's a chronic and untreatable neurodegenerative disease with irreversible progression and has important social and economic implications in terms of direct medical and social care costs. Despite prolonged and expensive efforts employed by the scientific community over the last few decades, no effective treatments are still available for patients, and the development of disease-modifying drugs is now a really urgent need. The recent failure of clinical trials based on the immunotherapeutic approach against amyloid- $\beta(A \beta)$ protein questioned the validity of the "amyloid cascade hypothesis" as the molecular machinery causing the disease. Indeed, most attempts to design effective treatments for $A D$ have been based until now on molecular targets suggested to be implicated in $\mathrm{AD}$ pathogenesis by the amyloid cascade hypothesis. However, mounting evidence from scientific literature supports the view of $A D$ as a multifactorial disease that results from the concomitant action of multiple molecular players. This view, together with the lack of success of the disease-modifying single-target approaches, strongly suggests that $A D$ drug design needs to be shifted towards multi-targeted compounds or drug combinations acting synergistically on the main core features of disease pathogenesis. The discovery of drug candidates targeting multiple factors involved in AD would greatly improve drug development. So, it is reasonable that upcoming strategies for the design of preventive and/or therapeutic agents for $A D$ point to a multi-pronged approach including more than one druggable target to definitely defeat the disease.

Keywords: Alzheimer, amyloid-beta, multi-target, tau, amyloid cascade hypothesis, amyloid precursor protein, secretase, biometal ions

\section{INTRODUCTION}

Alzheimer's disease (AD) is a multifactorial neurodegenerative disorder characterized by progressive loss of neurons which may induce a decline in learning ability, loss of memory and impairment of other cognitive functions (Lane et al., 2018). It is the most common cause of dementia in the elderly (Goedert and Spillantini, 2006). Nearly 44 million people worldwide suffer from dementia and the number of patients is likely to reach 135 million in 2050; more than half of them will be AD cases (Prince et al., 2013). No new drugs have been approved for AD during the past 16 years and the available medications have very low impact on the disease course (Cacabelos, 2018), and even increase the costs for the care of AD patients by prolonging the length of a person's "stay" in the Mild, Moderate, or Severe stage of disease (Cimler et al., 2019). For these reasons, AD is now recognized by the World Health Organization as a global public health priority. 
Despite large gains in our understanding of $\mathrm{AD}$ pathogenesis, no disease-modifying treatments are still available for patients. Huge data reported in literature suggest that multiple factors such as amyloid- $\beta$ (A $\beta)$ assemblies, tau-protein aggregation and hyperphosphorylation, low levels of acetylcholine (ACh), mitochondrial dysfunction, oxidative stress, inflammation and dyshomeostasis of biometals might be actively involved in $\mathrm{AD}$ (Querfurth and LaFerla, 2010; Pfaender and Grabrucker, 2014; Stancu et al., 2014; Selkoe and Hardy, 2016; Wilkins and Swerdlow, 2016; Hardy, 2017). Over the years, the amyloid cascade hypothesis emerged as the dominant model of $\mathrm{AD}$ pathogenesis and is still driving the development of potential treatments targeting the main molecular players of AD (Walker et al., 2005; Chakraborty, 2017).

The recent failure of clinical trials based on monoclonal antibodies (mAbs) against $\mathrm{A} \beta$ imposes urgent dilemmas on the interpretation of mechanistic studies and leads us to a crucial crossroad among the hypotheses on $\mathrm{AD}$ pathogenesis and to a revision of strategies employed until now for the design of efficient treatments.

In this review article, we summarize the reasons for the ineffectiveness of the main experimental strategies targeting the molecular pathways suggested to be crucial for the disease, and highlight the urgent need for a radical change in the therapeutic approaches to AD. These approaches, in our opinion, should definitely point to a synergic strategy based on the concomitant use of multiple drugs or of a single multi-target compound to tackle the most relevant events in the molecular machinery that causes the onset of $\mathrm{AD}$ and its progression.

\section{ON THE SIDE OF AMYLOID CASCADE HYPOTHESIS OR BEYOND IT?}

$\mathrm{AD}$ pathophysiological hallmarks include $\mathrm{A} \beta$ plaques and neurofibrillary tangles (NFTs), which predominantly aggregate in the hippocampus and neocortex (Hyman et al., 2012). A $\beta$ plaque deposition is associated with toxic soluble oligomers as well as eventual insoluble neuritic plaques (Hardy and Selkoe, 2002). For 25 years, the amyloid cascade hypothesis dominated the research on this disease (Hardy and Higgins, 1992; Selkoe and Hardy, 2016; Behl and Ziegler, 2017). It represented the almost exclusive source of the molecular targets for therapeutic strategies in $\mathrm{AD}$ and is supported by a long series of data reported in scientific literature during the last decades. The milestones in favor of this theory include genetic issues mainly provided by the discovery of pathogenic and protective mutations in the Amyloid Precursor Protein (APP; Selkoe, 1997; Di Fede et al., 2009; Jonsson et al., 2012; Hartley et al., 2015) and presenilin genes (Selkoe and Hardy, 2016), and the existence of polymorphisms in $A p o E$ and other recently discovered genes modulating the risk of developing $\mathrm{AD}$ (Liao et al., 2017; Kunkle et al., 2019). Additional evidence comes from mechanistic, neuropathological and imaging studies indicating $\mathrm{A} \beta$ oligomers and hyperphosphorylated tau as key players in disease pathogenesis (Goedert and Spillantini, 2006; Wang et al., 2013; Selkoe and Hardy, 2016). However, the approaches against amyloid cascade players explored until now in clinical trials were unsatisfactory, despite promising results in the preclinical phases of their development (Pinheiro and Faustino, 2019).

\section{Immunotherapy Against $\mathbf{A} \boldsymbol{\beta}$}

The strategy based on passive immunotherapy with anti$\mathrm{A} \beta$ antibodies followed the perception that administering targeted antibodies is more effective than trying to induce their production in vivo. However, the first approaches based on passive immunotherapy against $A \beta$ were carried out by using nonprotective mAbs against the $A \beta \mathrm{N}$-terminal domain $(\mathrm{A} \beta 1-15)$ that causes the release of cytotoxic $\mathrm{A} \beta$ oligomers which are immobilized as plaques, an event known as "dust raising effect" (Liu et al., 2015). Actually, most studies suggest that the immunogens needed to induce a protective immunity are the soluble $A \beta$ oligomers, rather than either monomeric $\mathrm{A} \beta$ or plaques (Selkoe and Hardy, 2016) and that the critical epitopes are conformational rather than linear (Wisniewski and Drummond, 2016). However, clinical trials with antibodies like aducanumab, which differ from previous mAbs targeting monomeric $\mathrm{A} \beta$, in that they recognize soluble cytotoxic $A \beta$ protofibrils and oligomers provided negative results too. A possible explanation for this lack of efficacy may derive from the observation that almost all immunotherapeutic approaches against $A \beta$ used the wrong $A \beta$-derived antigen, i.e., $A \beta 1-15$, combined with proinflammatory adjuvants, e.g., QS-21 and CpG oligodeoxynucleotides, which elicited an undesirable pro-inflammatory immunity (Th1/Th17) rather than the required anti-inflammatory one (Th2; Marciani, 2015). In this view, the discrepancies between preclinical and clinical results may be explained by the fact that transgenic animals are more resilient than humans to the side effects of pro-inflammatory adjuvants. The aducanumab-based protocol is associated with a prevalent but not exclusive trigger of Th2-mediated immunity (Marciani, 2015). This could explain its better-but still unclear-results in comparison with other immunotherapeutic protocols.

An additional problem increasing the uncertainties on drug design for $\mathrm{AD}$ is $\mathrm{A} \beta$ pleomorphism. Increasing evidence suggests that different $\mathrm{A} \beta$ assemblies may generate distinct $\mathrm{AD}$ phenotypes having different resistance to pharmacological treatments (Stöhr et al., 2014; Catania et al., 2015; Qiang et al., 2017; Rasmussen et al., 2017; Di Fede et al., 2018). Since different $\mathrm{mAbs}$ are directed against different conformational epitopes, it is reasonable to expect a synergism from the combined use of multiple antibodies (Marciani, 2019).

Hence, it is premature to conclude from the past failures of $\mathrm{AD}$ immunotherapy that $\mathrm{A} \beta$ is the wrong therapeutic target for vaccine development. These failures, at least in part, may be due to the use of inappropriate immunogens and adjuvants, incorrect timing of intervention, and/or wrong brain delivery strategies-only approximately $0.1 \%$ of mAbs cross the bloodbrain barrier (BBB; Lemere, 2013; van Dyck, 2018). Ongoing trials in $\mathrm{AD}$ preclinical phases to evaluate the safety, tolerability, and efficacy of mAbs in asymptomatic individuals at risk for developing AD based on PET-amyloid load, or with dominantly inherited forms of $\mathrm{AD}$, will provide useful information on the validity of such strategy (Bateman et al., 2017). 


\section{Other Therapeutic Strategies Targeting A $\beta$}

Beyond immunotherapy, $A \beta$-targeting compounds that have been tested or are currently under assessment include drugs interfering with APP processing-i.e., $\alpha$-secretase activators, $\beta$-secretase inhibitors, $\gamma$-secretase modulators-and inhibitors of $A \beta$ aggregation (Citron, 2010; Suzuki et al., 2017; Umar and Hoda, 2017), based on the view that an increased amyloid production favors the onset of $\mathrm{AD}$ in animal models and humans carrying genetic defects in the three causative genes associated with early-onset AD, i.e., APP, presenilin 1 and 2 (Selkoe, 1991; Hardy and Selkoe, 2002; Kunkle et al., 2019).

Activation of $\alpha$-secretase prevents the formation of toxic $A \beta$ peptides and promotes the secretion of neurotrophic sAPP $\alpha$ by cleaving APP within the A $\beta$ sequence (Beeg et al., 2016). A limited number of activators of $\alpha$-secretase reached clinical testing and displayed fewer side effects in comparison with $\beta$ - and $\gamma$-secretase inhibitors (GSIs) without showing efficacy on primary endpoints; some of them are currently under evaluation (Pinheiro and Faustino, 2019).

Inhibitors of $\beta$-secretase enzymes (BACE1 and BACE2) were proposed as disease-modifying drugs in $\mathrm{AD}$ following the preclinical observation that knockout mice do not develop cerebral amyloidosis (Dominguez et al., 2005). Nonetheless, most BACE inhibitors tested in controlled trials failed to prevent cognitive decline. This lack of efficacy has been attributed to their use in advanced stages of the disease (Pinheiro and Faustino, 2019). Actually, the only remaining trials with a BACE inhibitor (Elenbecestat) have been very recently halted for safety reasons ${ }^{1}$.

GSIs were developed to decrease $A \beta$ production by inhibiting intramembrane APP cleavage (He et al., 2010). However, they are associated with severe gastrointestinal/immunological side effects due to abnormal processing of the Notch 1 transmembrane receptor, which is also a substrate of $\gamma$-secretase, leading to increased risk of serious adverse events, including infections and skin cancers (Penninkilampi et al., 2016). These data shifted the interest of researchers to more selective GSIs (Teranishi et al., 2015) and modulators of $\gamma$-secretase (Xia, 2019) to overcome the side effects of first-generation GSIs. However, when tested in humans, even Notch-sparing GSIs failed to efficiently contrast the disease or their use was early terminated due to unacceptable side effects, especially on liver function (Doody et al., 2013; Pinheiro and Faustino, 2019).

Another anti-amyloid strategy is based on inhibitors of $A \beta$ aggregation. Two main classes of molecules were developed in the last decades against AD: (i) short synthetic $\beta$-sheet breaker peptides; and (ii) nonpeptidic small drugs. However, the former generally showed poor pharmacokinetic profile, including low solubility, poor oral bioavailability and BBB permeability and elicitation of adverse immunogenic/inflammatory responses (Re et al., 2010; Hamley, 2012), and have not yet been pursued in clinical trials (Pinheiro and Faustino, 2019), with some exceptions (Shea et al., 2019). The latter, which include naturally occurring flavonoids and polyphenols, $\omega-3$ polyunsaturated fatty acids (e.g., docosahexaenoic acid), natural organic dyes,

\footnotetext{
${ }^{1}$ https://www.alzforum.org/news/research-news/end-bace-inhibitorselenbecestat-trials-halted-amid-safety-concerns
}

some drugs (e.g., rifampicin and tetracycline antibiotics), ionic surfactants (e.g., sodium dodecyl sulfate and hexadecylNmethylpiperidinium bromide) and several sulfur-containing compounds (e.g., $\alpha$-lipoic acid and $N$-acetylcysteine) were reported to have some benefits in animal models (Ladiwala et al., 2011; Giorgetti et al., 2018) and in few clinical trials (Salloway et al., 2011; Cummings et al., 2016; Hey et al., 2018). Important limitations for the application of these group of compounds into clinical practice come from their nonspecific targets. The employment of computational approaches combined with biophysical/biochemical methods will likely help to clarify the mechanisms of interaction with targets of amyloid cascade, providing more effective drugs in the near future (Giorgetti et al., 2018).

A similar strategy based on the use of all-D peptides able to hinder $\mathrm{A} \beta$ nucleation/polymerization led recently to the design of an ongoing and promising trial (PRI-002 from PRIAVOID; Agerschou et al., 2019; Zhang T. et al., 2019).

$\mathrm{A}$ recent $\mathrm{A} \beta$-targeting approach was proposed by our group. It is based on the use of a naturally occurring genetic variant of $A \beta$, consisting of an alanine-to-valine substitution at position 2 of the $A \beta$ sequence $\left(A \beta_{A 2 V}\right)$. This variant-that in the homozygous state is pathogenic-is instead surprisingly protective against $A D$ in $A \beta_{A 2 V}$ heterozygous carriers (Di Fede et al., 2009; Diomede et al., 2014). The " $\mathrm{A} \beta_{\mathrm{A} 2 \mathrm{~V}}$-based strategy" has been tested in preclinical studies showing promising results in preventing $\mathrm{A} \beta$ aggregation and cerebral amyloid deposition, synaptic impairment and cognitive decline (Di Fede et al., 2012, 2016; Cimini et al., 2016).

\section{Other Approaches Based on Amyloid Cascade Targets}

Several studies in cell and animal models indicated that oligomeric, soluble $A \beta$ is the primary driving force of $A D$ pathogenesis but its main neurodegenerative changes are mediated at least partially by tau protein (Brandt and Bakota, 2017). Hence, tau and microtubules were emphasized as a target for therapeutic intervention in AD (Brandt and Bakota, 2017). The most promising tau-targeting strategies are inhibition of tau phosphorylation, proteolysis and aggregation, promotion of intracellular and extracellular tau clearance, prevention of tau spreading and stabilization of microtubules (Šimić et al., 2016). Initially, anti-tau therapies were based mainly on inhibition of kinases or tau aggregation or on stabilization of microtubules, but most of these approaches have been discontinued because of toxicity and/or lack of efficacy (Congdon and Sigurdsson, 2018). Immunotherapy against tau is now under evaluation in clinical trials as an approach potentially able to interfere with more than one of the above-cited mechanistic events (Hoskin et al., 2019). However, current tau-based immunotherapy programs are raising additional questions concerning the choice of the most efficient epitopes, the induction of extracellular vs. intracellular clearance of tau protein, the strength of the affinity of mAbs for tau, which can deeply modify the efficacy and safety of tau-targeting strategy (Golde, 2014; Pedersen and Sigurdsson, 2015; Sigurdsson, 2016). An alternative promising approach is the use of antisense oligonucleotides to decrease the expression 
of tau. This strategy is currently under evaluation in clinical trials for $\mathrm{AD}$ (DeVos et al., 2017).

\section{Alternative Pathogenic Hypotheses and Therapeutic Targets}

$\mathrm{AD}$ patients have lower levels of $\mathrm{ACh}$ and an impaired cholinergic transmission, resulting in learning and memory dysfunction. The possibility of modulation of these related events gave rise to the "Cholinergic Hypothesis" for $\mathrm{AD}$, which calls for enhancing cholinergic neurotransmission by the inhibition of the enzyme responsible for the metabolic breakdown of ACh (de Freitas Silva et al., 2018). Drugs currently used in the treatment of $\mathrm{AD}$ are mainly based on this evidence. They are acetylcholinesterase inhibitors: donepezil, rivastigmine, galantamine (just to enhance cholinergic neurotransmission), and the NMDAR antagonist memantine (addressing dysfunctional glutamatergic neurotransmission). The latter found its rationale on evidence that most neurotoxic events in $\mathrm{AD}$ are mediated by glutamate receptors (Zhang et al., 2016). However, these drugs do not prevent or reverse the progression of the disease, providing only symptomatic relief and/or short-term benefits (Pinheiro and Faustino, 2019).

Neuroinflammation is a common feature of $\mathrm{AD}$ and other neurodegenerative diseases, and increasing evidence indicates that neuroinflammatory processes contribute to and modulate disease pathogenesis (Walter et al., 2017; Heneka et al., 2014). However, thus far, most experimental single-target drugs that are effective in animal models were not effective in $\mathrm{AD}$ clinical trials (Van der Schyf, 2011). This failure was ascribed to problems in reaching or binding to the human targets in vivo, delayed timing of intervention, inappropriate design of clinical trial or lack of strength of the drug-target interaction to reduce the signs and symptoms of the disease (Wenzel and Klegeris, 2018). Another complication of the anti-inflammatory approach comes from the unclear role of microglia and the double-edged sword of its interplay with $A \beta$ since both detrimental and safe effects are attributed to microglial cells in $\mathrm{AD}$ brains (Hansen et al., 2018; McQuade and Blurton-Jones, 2019). However, a multitarget approach against players of neuroinflammation-such as Cathepsin B, mitogen-activated protein kinases (MAPKs), monoacylglycerol lipase-was proposed as a disease-modifying therapy for $\mathrm{AD}$ and could theoretically overcome the limitations of the neuroinflammatory single target strategies (Jalili-Baleh et al., 2018; Wenzel and Klegeris, 2018).

The involvement of "biometals" in AD pathogenesis was indicated by studies showing that metal ions, namely $\mathrm{Cu}^{2+}$, $\mathrm{Zn}^{2+}$ and $\mathrm{Fe}^{3+}$, are known to promote the oligomerization of monomeric $\mathrm{A} \beta$ and to induce oxidative stress in $\mathrm{AD}$ brain (Grasso et al., 2012). So, metal chelation was employed as a strategy for the development of $\mathrm{AD}$ therapeutics based on its ability to reverse metal-induced $\mathrm{A} \beta$ aggregation in human brain tissue (Ayton et al., 2015). The potential for metal-based drug therapy is likely not yet fully explored by clinical trials, despite some encouraging results in humans showing mitigation of cognitive dysfunctions in AD patients (Faux et al., 2010).

It is well recognized that oxidative stress can contribute to aging-related neurodegenerative diseases including $\mathrm{AD}$. It is also well accepted that natural compounds and minerals are powerful antioxidants that offer health benefits against several different degenerative disorders, hence the increasing interest in developing anti-oxidative therapeutics for AD. Unfortunately, there are still contradictory and inconsistent reports on the possible benefits of anti-oxidative supplements (Poprac et al., 2017; Thapa and Carroll, 2017).

\section{MULTI-TARGET APPROACHES FOR AD}

Despite extensive efforts and investments, the $\mathrm{AD}$ scientific community has been frustrated by the lack of clinical outcomes and research breakthroughs that have been translatable to clinical application. However, important suggestions come from the critical analysis of such flop. Several features contributed to the failure of experimental drugs against $\mathrm{AD}$, including inadequate preclinical data, poor BBB penetration, low therapeutic window, inadequate patient selection and/or inaccurate diagnosis (Doig et al., 2017; Pinheiro and Faustino, 2019). The timing of an $\mathrm{A} \beta$ - or tau-targeted intervention has proven critical for clinical response since once $A \beta$-induced synaptic dysfunction and extensive neurodegeneration occur, they can no longer be reversed by simply reducing brain amyloid burden (Cao et al., 2018). This paradigm has shifted clinical trials from late clinical $\mathrm{AD}$ dementia to the early, asymptomatic stages of the disease (Pinheiro and Faustino, 2019). However, this time shift is likely still not enough.

In our opinion, amyloid cascade should be interpreted not as a linear chain of consequent steps but rather as a multi-branched network of events triggered by the formation of soluble $A \beta$ oligomers and each characterized by self-progression (Figure 1). In this view, to substantially interfere with the molecular machinery initiated by $A \beta$, it is far not enough to stop the amyloid pathology-unless this is achieved very early, in the preclinical phase of the disease. More chances of being successful may come from combined approaches targeting multiple molecular players of the amyloid cascade.

Current drug design strategies are based on "one drug-one target" paradigm (Schneider et al., 2014), that until now failed to provide effective treatments against $\mathrm{AD}$, due to the multifactorial nature of the disease (Kumar et al., 2018; Ibrahim and Gabr, 2019). Multi-target approaches for the rational design of novel drug candidates, also called multitarget-directed ligands (MTDL) strategies, have been used to develop a variety of hybrid compounds capable to act simultaneously in diverse biological targets (Viegas-Junior et al., 2007; Hughes et al., 2016; Agatonovic-Kustrin et al., 2018; Batool et al., 2018; de Freitas Silva et al., 2018; González et al., 2019). They are mostly driven by computational drug designing methods capable of assisting drug discovery (Anastasio, 2015). However, complementary tools and expertise are requested to optimize multi-target drug development (Kumar et al., 2018), including more appropriate animal models, which can fully recapitulate the molecular events of amyloid cascade (Moreno-Gonzalez and Soto, 2012; Drummond and Wisniewski, 2017).

The design of MTDLs is at the beginning of its history even if, in the recent years, several compounds 


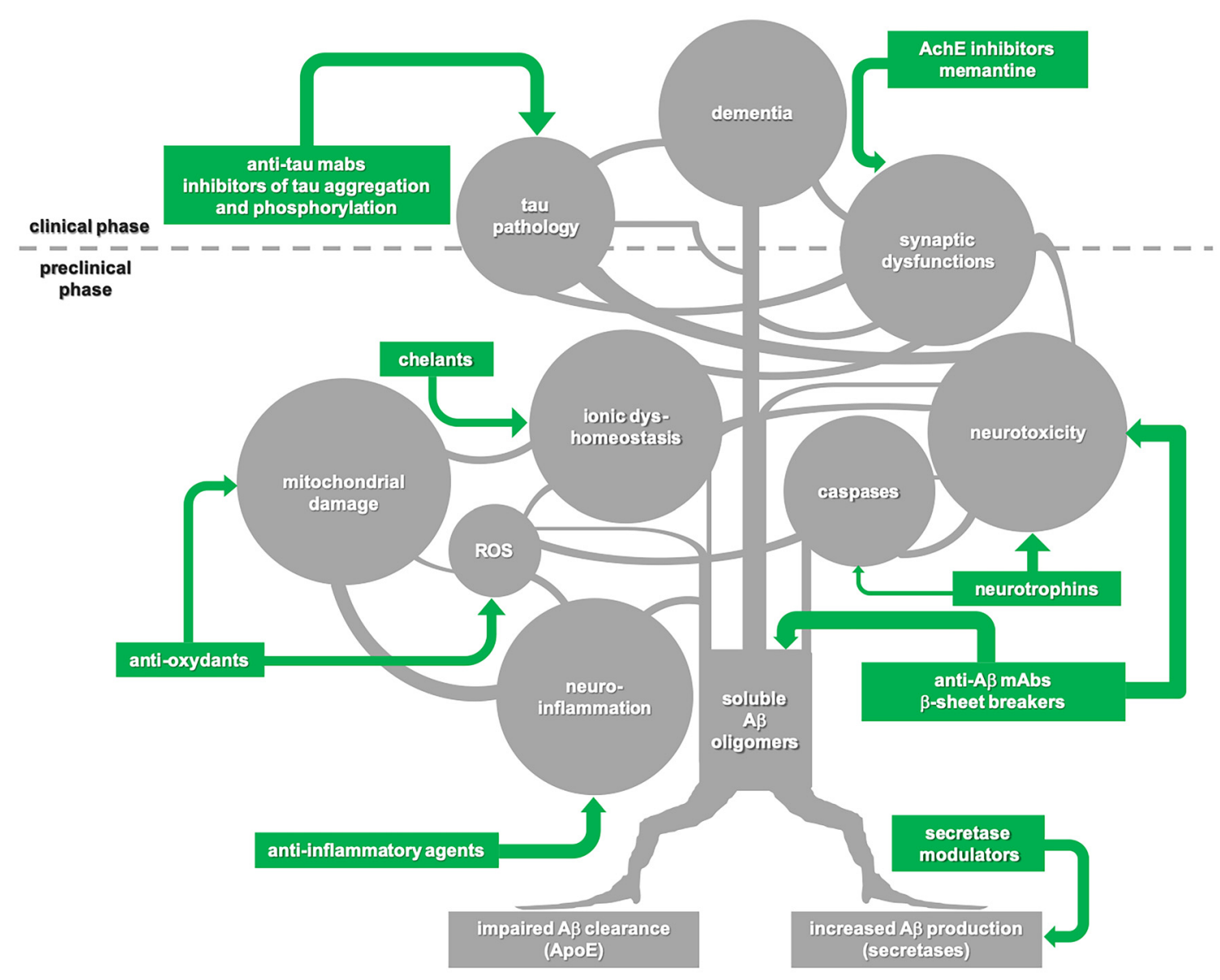

FIGURE 1 | Schematic illustration of the "amyloid cascade hypothesis" that is viewed here as a tricky multi-branched process where different molecular events evolve autonomously, once triggered by the initial generation of soluble amyloid- $\beta(A \beta)$ oligomers. Tau-mediated neuronal damage and neuronal toxicity induced by activation of caspases and neuroinflammation, oxidative stress with mitochondrial dysfunction, dyshomeostasis of biometals and synaptic failure are self-dependent players which synergistically sustain the onset and progression of the disease. Following this view, disease-modifying treatments for Alzheimer's disease (AD) should be based on combined approaches against multiple targets to be successful. Moreover, taking into account that the majority of the key events in the cascade begin during the preclinical phase of the disease, a preventive multi-target strategy promises to be much more effective in comparison with late, single-target approaches.

(Viegas et al., 2005; Guzior et al., 2015; Romero and MarcoContelles, 2017; Umar and Hoda, 2017; Zhang P. et al., 2019) retaining at the same time anti-aggregation properties against $\mathrm{A} \beta$ and cholinesterase inhibition activity or coupling modulation of serotonin and ACh pathways with the release of soluble forms of $\operatorname{APP}(\operatorname{sAP} \alpha)$-having neurotrophic properties-or combining chemical structures able to interact with monoamine oxidase and amyloid-binding alcohol dehydrogenase (Hroch et al., 2017) have been developed and tested in preclinical studies showing promising results (Lecoutey et al., 2014; Rochais et al., 2015; Hatat et al., 2019). However, until now no multitarget compounds have been successfully translated to the clinical context.

\section{CONCLUDING REMARKS}

In conclusion, the multifactorial nature of $\mathrm{AD}$ pathophysiology supports the design of druggable compounds which can be translated into new effective and well-tolerated drugs able to interact synergistically with different targets, modulating different interconnected molecular pathways related to the disease onset and progression. There is increasing evidence in the literature in favor of the beneficial role of multi-target strategy in the cure of multifactorial diseases. In this context, several substances with multi-target activity were discovered and several of them showed interesting pharmacological profiles, making them possible drug candidates (de Freitas Silva et al., 2018), even if their development can be slowed down by the fact that MTDLs may require different concentrations to tackle different pathways. However, this disadvantage can be overcome by the combined use of multiple drugs rather than a single multitarget drug. To our knowledge, combined multi-target diseasemodifying treatments against the most relevant molecular players of $\mathrm{AD}$ have not yet tested in controlled clinical trials. It is time for a coordinated multi-target attack on amyloid- $\beta$ cascade in $\mathrm{AD}$. 


\section{AUTHOR CONTRIBUTIONS}

MC wrote the manuscript and designed the figure. GF revised and edited the manuscript. GG, MS and FT supervised the work.

\section{REFERENCES}

Agatonovic-Kustrin, S., Kettle, C., and Morton, D. W. (2018). A molecular approach in drug development for Alzheimer's disease. Biomed. Pharmacother. 106, 553-565. doi: 10.1016/j.biopha.2018.06.147

Agerschou, E. D., Flagmeier, P., Saridaki, T., Galvagnion, C., Komnig, D., Heid, L., et al. (2019). An engineered monomer binding-protein for $\alpha$-synuclein efficiently inhibits the proliferation of amyloid fibrils. Elife 8:e46112. doi: 10.7554/eLife.46112

Anastasio, T. J. (2015). Computational identification of potential multi-drug combinations for reduction of microglial inflammation in Alzheimer disease. Front. Pharmacol. 6:116. doi: 10.3389/fphar.2015.00116

Ayton, S., Lei, P., and Bush, A. I. (2015). Biometals and their therapeutic implications in Alzheimer's disease. Neurotherapeutics 12, 109-120. doi: 10.1007/s13311-014-0312-z

Bateman, R. J., Benzinger, T. L., Berry, S., Clifford, D. B., Duggan, C., Fagan, A. M., et al. (2017). The DIAN-TU next generation Alzheimer's prevention trial: adaptive design and disease progression model. Alzheimers Dement. 13, 8-19. doi: 10.1016/j.jalz.2016.07.005

Batool, A., Kamal, M. A., Rizvi, S. M. D., and Rashid, S. (2018). Topical discoveries on multi-target approach to manage Alzheimer's disease. Curr. Drug Metab. 19, 704-713. doi: 10.2174/1389200219666180305152553

Beeg, M., Stravalaci, M., Romeo, M., Carrá, A. D., Cagnotto, A., Rossi, A., et al. (2016). Clusterin binds to A $\beta 1-42$ oligomers with high affinity and interferes with peptide aggregation by inhibiting primary and secondary nucleation. J. Biol. Chem. 291, 6958-6966. doi: 10.1074/jbc.M115. 689539

Behl, C., and Ziegler, C. (2017). Beyond amyloid-widening the view on Alzheimer's disease. J. Neurochem. 143, 394-395. doi: 10.1111/jnc.14137

Brandt, R., and Bakota, L. (2017). Microtubule dynamics and the neurodegenerative triad of Alzheimer's disease: the hidden connection. J. Neurochem. 143, 409-417. doi: 10.1111/jnc.14011

Cacabelos, R. (2018). Have there been improvements in Alzheimer's disease drug discovery over the past 5 years? Expert Opin. Drug Discov. 13, 523-538. doi: 10.1080/17460441.2018.1457645

Cao, J., Hou, J., Ping, J., and Cai, D. (2018). Advances in developing novel therapeutic strategies for Alzheimer's disease. Mol. Neurodegener. 13:64. doi: 10.1186/s13024-018-0299-8

Catania, M., Di Fede, G., Tonoli, E., Benussi, L., Pasquali, C., Giaccone, G., et al. (2015). Mirror image of the amyloid- $\beta$ species in cerebrospinal fluid and cerebral amyloid in Alzheimer's disease. J. Alzheimers Dis. 47, 877-881. doi: 10.3233/JAD-150179

Chakraborty, S. (2017). Multi-potent natural scaffolds targeting amyloid cascade: in search of Alzheimer's disease therapeutics. Curr. Top. Med. Chem. 17, 3336-3348. doi: 10.2174/1568026618666180116122921

Cimini, S., Sclip, A., Mancini, S., Colombo, L., Messa, M., Cagnotto, A., et al. (2016). The cell-permeable A $\beta 1-6_{\mathrm{A} 2 \mathrm{~V}} \mathrm{TAT}(\mathrm{D})$ peptide reverts synaptopathy

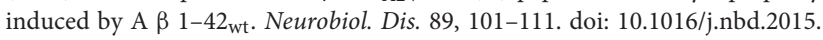
12.013

Cimler, R., Maresova, P., Kuhnova, J., and Kuca, K. (2019). Predictions of Alzheimer's disease treatment and care costs in European countries. PLoS One 14:e0210958. doi: 10.1371/journal.pone.0210958

Citron, M. (2010). Alzheimer's disease: strategies for disease modification. Nat. Rev. Drug Discov. 9, 387-398. doi: 10.1038/nrd2896

Congdon, E. E., and Sigurdsson, E. M. (2018). Tau-targeting therapies for Alzheimer disease. Nat. Rev. Neurol. 14, 399-415. doi: 10.1038/s41582-0180013-z

Cummings, J. L., Zhong, K., Kinney, J. W., Heaney, C., Moll-Tudla, J., Joshi, A., et al. (2016). Double-blind, placebo-controlled, proof-of-concept trial of bexarotene Xin moderate Alzheimer's disease. Alzheimers Res. Ther. 8:4. doi: 10.1186/s13195-016-0173-2

\section{FUNDING}

This work was supported by the Current Research funded by the Italian Ministry of Health (Ministero della Salute) to GF and MC.

de Freitas Silva, M., Dias, K. S. T., Gontijo, V. S., Ortiz, C. J. C., and Viegas, C. Jr. (2018). Multi-target directed drugs as a modern approach for drug design towards Alzheimer's disease: an update. Curr. Med. Chem. 25, 3491-3525. doi: 10.2174/0929867325666180111101843

DeVos, S. L., Miller, R. L., Schoch, K. M., Holmes, B. B., Kebodeaux, C. S., Wegener, A. J., et al. (2017). Tau reduction prevents neuronal loss and reverses pathological tau deposition and seeding in mice with tauopathy. Sci. Transl. Med. 9:eaag0481. doi: 10.1126/scitranslmed.aag0481

Di Fede, G., Catania, M., Maderna, E., Ghidoni, R., Benussi, L., Tonoli, E., et al. (2018). Molecular subtypes of Alzheimer's disease. Sci. Rep. 8:3269. doi: 10.1038/s41598-018-21641-1

Di Fede, G., Catania, M., Maderna, E., Morbin, M., Moda, F., Colombo, L., et al. (2016). Tackling amyloidogenesis in Alzheimer's disease with A2V variants of amyloid- $\beta$. Sci. Rep. 6:20949. doi: 10.1038/srep20949

Di Fede, G., Catania, M., Morbin, M., Giaccone, G., Moro, M. L., Ghidoni, R., et al. (2012). Good gene, bad gene: new APP variant may be both. Prog. Neurobiol. 99, 281-292. doi: 10.1016/j.pneurobio.2012.06.004

Di Fede, G., Catania, M., Morbin, M., Rossi, G., Suardi, S., Mazzoleni, G., et al. (2009). A recessive mutation in the APP gene with dominant-negative effect on amyloidogenesis. Science 323, 1473-1477. doi: 10.1126/science.1168979

Diomede, L., Di Fede, G., Romeo, M., Bagnati, R., Ghidoni, R., Fiordaliso, F., et al. (2014). Expression of $\mathrm{A} 2 \mathrm{~V}$-mutated $\mathrm{A} \beta$ in Caenorhabditis elegans results in oligomer formation and toxicity. Neurobiol. Dis. 62, 521-532. doi: 10.1016/j. nbd.2013.10.024

Doig, A. J., Del Castillo-Frias, M. P., Berthoumieu, O., Tarus, B., NasicaLabouze, J., Sterpone, F., et al. (2017). Why is research on amyloid- $\beta$ failing to give new drugs for Alzheimer's disease? ACS Chem. Neurosci. 8, 1435-1437. doi: 10.1021/acschemneuro.7b00188

Dominguez, D., Tournoy, J., Hartmann, D., Huth, T., Cryns, K., Deforce, S., et al. (2005). Phenotypic and biochemical analyses of BACE1- and BACE2-deficient mice. J. Biol. Chem. 280, 30797-30806. doi: 10.1074/jbc.m505249200

Doody, R. S., Raman, R., Farlow, M., Iwatsubo, T., Vellas, B., Joffe, S., et al. (2013). A phase 3 trial of semagacestat for treatment of Alzheimer's disease. N. Engl. J. Med. 369, 341-350. doi: 10.1056/NEJMoa1210951

Drummond, E., and Wisniewski, T. (2017). Alzheimer's disease: experimental models and reality. Acta Neuropathol. 133, 155-175. doi: 10.1007/s00401-0161662-x

Faux, N. G., Ritchie, C. W., Gunn, A., Rembach, A., Tsatsanis, A., Bedo, J., et al. (2010). PBT2 rapidly improves cognition in Alzheimer's disease: additional phase II analyses. J. Alzheimers Dis. 20, 509-516. doi: 10.3233/jad-20 $10-1390$

Giorgetti, S., Greco, C., Tortora, P., and Aprile, F. A. (2018). Targeting amyloid aggregation: an overview of strategies and mechanisms. Int. J. Mol. Sci. 19:E2677. doi: 10.3390/ijms19092677

Goedert, M., and Spillantini, M. G. (2006). A century of Alzheimer's disease. Science 314, 777-781. doi: 10.1126/science.1132814

Golde, T. E. (2014). Open questions for Alzheimer's disease immunotherapy. Alzheimers Res. Ther. 6:3. doi: 10.1186/alzrt233

González, J. F., Alcántara, A. R., Doadrio, A. L., and Sánchez-Montero, J. M. (2019). Developments with multi-target drugs for Alzheimer's disease: an overview of the current discovery approaches. Expert. Opin. Drug Discov. 14, 879-891. doi: 10.1080/17460441.2019.1623201

Grasso, G., Giuffrida, M. L., and Rizzarelli, E. (2012). Metallostasis and amyloid $\beta$-degrading enzymes. Metallomics 4, 937-949. doi: 10.1039/c2mt20105d

Guzior, N., Wieckowska, A., Panek, D., and Malawska, B. (2015). Recent development of multifunctional agents as potential drug candidates for the treatment of Alzheimer's disease. Curr. Med. Chem. 22, 373-404. doi: 10.2174/0929867321666141106122628

Hamley, I. W. (2012). The amyloid- $\beta$ peptide: a chemist's perspective. Role in Alzheimer's and fibrillization. Chem. Rev. 112, 5147-5192. doi: $10.1021 / \mathrm{cr} 3000994$ 
Hansen, D. V., Hanson, J. E., and Sheng, M. (2018). Microglia in Alzheimer's disease. J. Cell Biol. 217, 459-472. doi: 10.1083/jcb.201709069

Hardy, J. (2017). The discovery of Alzheimer-causing mutations in the APP gene and the formulation of the "amyloid cascade hypothesis". FEBS J. 284, 1040-1044. doi: 10.1111/febs.14004

Hardy, J. A., and Higgins, G. A. (1992). Alzheimer's disease: the amyloid cascade hypothesis. Science 256, 184-185. doi: 10.1126/science.1566067

Hardy, J., and Selkoe, D. J. (2002). The amyloid hypothesis of Alzheimer's disease: progress and problems on the road to therapeutics. Science 297, 353-356. doi: $10.1126 /$ science. 1072994

Hartley, D., Blumenthal, T., Carrillo, M., DiPaolo, G., Esralew, L., Gardiner, K., et al. (2015). Down syndrome and Alzheimer's disease: common pathways, common goals. Alzheimers Dement. 11, 700-709. doi: 10.1016/j.jalz.2014. 10.007

Hatat, B., Yahiaoui, S., Lecoutey, C., Davis, A., Freret, T., Boulouard, M., et al. (2019). A novel in vivo anti-amnesic agent, specially designed to express both acetylcholinesterase (AChE) inhibitory, serotonergic subtype 4 receptor $\left(5-\mathrm{HT}_{4} \mathrm{R}\right)$ agonist and serotonergic subtype 6 receptor $\left(5-\mathrm{HT}_{6} \mathrm{R}\right)$ inverse agonist activities, with a potential interest against Alzheimer's disease. Front. Aging Neurosci. 11:148. doi: 10.3389/fnagi.2019.00148

He, G., Luo, W., Li, P., Remmers, C., Netzer, W. J., Hendrick, J., et al. (2010). $\gamma$-secretase activating protein is a therapeutic target for Alzheimer's disease. Nature 467, 95-98. doi: 10.1038/nature09325

Heneka, M. T., Kummer, M. P., and Latz, E. (2014). Innate immune activation in neurodegenerative disease. Nat. Rev. Immunol. 14, 463-477. doi: $10.1038 /$ nri3705

Hey, J. A., Kocis, P., Hort, J., Abushakra, S., Power, A., Vyhnalek, M., et al. (2018). Discovery and identification of an endogenous metabolite of tramiprosate and its prodrug ALZ-801 that inhibits $\beta$-amyloid oligomer formation in the human brain. CNS Drugs 32, 849-861. doi: 10.1007/s40263-018-0554-0

Hoskin, J. L., Sabbagh, M. N., Al-Hasan, Y., and Decourt, B. (2019). Tau immunotherapies for Alzheimer's disease. Expert Opin. Investig. Drugs 28, 545-554. doi: 10.1080/13543784.2019.1619694

Hroch, L., Guest, P., Benek, O., Soukup, O., Janockova, J., Dolezal, R., et al. (2017). Synthesis and evaluation of frentizole-based indolyl thiourea analogues as MAO/ABAD inhibitors for Alzheimer's disease treatment. Bioorg. Med. Chem. 25, 1143-1152. doi: 10.1016/j.bmc.2016.12.029

Hughes, R. E., Nikolic, K., and Ramsay, R. R. (2016). One for all? Hitting multiple Alzheimer's disease targets with one drug. Front. Neurosci. 10:177. doi: 10.3389/fnins.2016.00177

Hyman, B. T., Phelps, C. H., Beach, T. G., Bigio, E. H., Cairns, N. J., Carrillo, M. C., et al. (2012). National institute on aging-Alzheimer's association guidelines for the neuropathologic assessment of Alzheimer's disease. Alzheimers Dement. 8, 1-13. doi: 10.1016/j.jalz.2011.10.007

Ibrahim, M. M., and Gabr, M. T. (2019). Multitarget therapeutic strategies for Alzheimer's disease. Neural Regen. Res. 14, 437-440. doi: 10.4103/1673-5374. 245463

Jalili-Baleh, L., Forootanfar, H., Kucukkilinc, T. T., Nadri, H., Abdolahi, Z., Ameri, A., et al. (2018). Design, synthesis and evaluation of novel multi-targetdirected ligands for treatment of Alzheimer's disease based on coumarin and lipoic acid scaffolds. Eur. J. Med. Chem. 152, 600-614. doi: 10.1016/j.ejmech. 2018.04.058

Jonsson, T., Atwal, J. K., Steinberg, S., Snaedal, J., Jonsson, P. V., Bjornsson, S., et al. (2012). A mutation in APP protects against Alzheimer's disease and age-related cognitive decline. Nature 488, 96-99. doi: 10.1038/nature11283

Kumar, A., Tiwari, A., and Sharma, A. (2018). Changing paradigm from one target one ligand towards multi-target directed ligand design for key drug targets of Alzheimer disease: an important role of in silico methods in multi-target directed ligands design. Curr. Neuropharmacol. 16, 726-739. doi: 10.2174/1570159x16666180315141643

Kunkle, B. W., Grenier-Boley, B., Sims, R., Bis, J. C., Damotte, V., Naj, A. C., et al. (2019). Genetic meta-analysis of diagnosed Alzheimer's disease identifies new risk loci and implicates $A \beta$, tau, immunity and lipid processing. Nat. Genet. 51, 414-430. doi: 10.1038/s41588-019-0358-2

Ladiwala, A. R., Dordick, J. S., and Tessier, P. M. (2011). Aromatic small molecules remodel toxic soluble oligomers of amyloid- $\beta$ through three independent pathways. J. Biol. Chem. 286, 3209-3218. doi: 10.1074/jbc.M110. 173856
Lane, C. A., Hardy, J., and Schott, J. M. (2018). Alzheimer's disease. Eur. J. Neurol. 25, 59-70. doi: 10.1111/ene.13439

Lecoutey, C., Hedou, D., Freret, T., Giannoni, P., Gaven, F., Since, M., et al. (2014). Design of donecopride, a dual serotonin subtype 4 receptor agonist/acetylcholinesterase inhibitor with potential interest for Alzheimer's disease treatment. Proc. Natl. Acad. Sci. U S A 111, E3825-E3830. doi: 10.1073/pnas. 1410315111

Lemere, C. A. (2013). Immunotherapy for Alzheimer's disease: hoops and hurdles. Mol. Neurodegener. 8:36. doi: 10.1186/1750-1326-8-36

Liao, F., Yoon, H., and Kim, J. (2017). Apolipoprotein E metabolism and functions in brain and its role in Alzheimer's disease. Curr. Opin. Lipidol. 28, 60-67. doi: 10.1097/MOL.0000000000000383

Liu, Y. H., Bu, X. L., Liang, C. R., Wang, Y. R., Zhang, T., Jiao, S. S., et al. (2015). An N-terminal antibody promotes the transformation of amyloid fibrils into oligomers and enhances the neurotoxicity of amyloid- $\beta$ : the dust-raising effect. J. Neuroinflammation 12:153. doi: 10.1186/s12974-015-0379-4

Marciani, D. J. (2015). Alzheimer's disease vaccine development: a new strategy focusing on immune modulation. J. Neuroimmunol. 287, 54-63. doi: 10.1016/j. jneuroim.2015.08.008

Marciani, D. J. (2019). Promising results from Alzheimer's disease passive immunotherapy support the development of a preventive vaccine. Research 2019:5341375. doi: 10.34133/2019/5341375

McQuade, A., and Blurton-Jones, M. (2019). Microglia in Alzheimer's disease: exploring how genetics and phenotype influence risk. J. Mol. Biol. 431, 1805-1817. doi: 10.1016/j.jmb.2019.01.045

Moreno-Gonzalez, I., and Soto, C. (2012). Natural animal models of 1 neurodegenerative protein misfolding diseases. Curr. Pharm. Des. 18, 1148-1158. doi: 10.2174/138161212799315768

Pedersen, J. T., and Sigurdsson, E. M. (2015). Tau immunotherapy for Alzheimer's disease. Trends Mol. Med. 21, 394-402. doi: 10.1016/j.molmed.2015.03.003

Penninkilampi, R., Brothers, H. M., and Eslick, G. D. (2016). Pharmacological agents targeting $\gamma$-secretase increase risk of cancer and cognitive decline in Alzheimer's disease patients: a systematic review and meta-analysis. J. Alzheimers Dis. 53, 1395-1404. doi: 10.3233/jad-160275

Pfaender, S., and Grabrucker, A. M. (2014). Characterization of biometal profiles in neurological disorders. Metallomics 6, 960-977. doi: 10.1039/c4mt00008k

Pinheiro, L., and Faustino, C. (2019). Therapeutic strategies targeting amyloid- $\beta$ in Alzheimer's disease. Curr. Alzheimer Res. 16, 418-452. doi: 10.2174/1567205016666190321163438

Poprac, P., Jomova, K., Simunkova, M., Kollar, V., Rhodes, C. J., and Valko, M. (2017). Targeting free radicals in oxidative stress-related human diseases. Trends Pharmacol. Sci. 38, 592-607. doi: 10.1016/j.tips.2017.04.005

Prince, M., Guerchet, M., and Prina, M. (2013). Alzheimer's Disease International. Policy Brief for Heads of Government: the Global Impact of Dementia. London: Alzheimer's Disease International.

Qiang, W., Yau, W. M., Lu, J. X., Collinge, J., and Tycko, R. (2017). Structural variation in amyloid- $\beta$ fibrils from Alzheimer's disease clinical subtypes. Nature 541, 217-221. doi: 10.1038/nature20814

Querfurth, H. W., and LaFerla, F. M. (2010). Alzheimer's disease. N. Engl. J. Med. 362, 329-344. doi: 10.1056/NEJMra0909142

Rasmussen, J., Mahler, J., Beschorner, N., Kaeser, S. A., Häsler, L. M., Baumann, F., et al. (2017). Amyloid polymorphisms constitute distinct clouds of conformational variants in different etiological subtypes of Alzheimer's disease. Proc. Natl. Acad. Sci. U S A 114, 13018-13023. doi: 10.1073/pnas. 1713215114

Re, F., Airoldi, C., Zona, C., Masserini, M., La Ferla, B., Quattrocchi, N., et al. (2010). $\beta$-amyloid aggregation inhibitors: small molecules as candidate drugs for therapy of Alzheimer's disease. Curr. Med. Chem. 17, 2990-3006. doi: $10.2174 / 092986710791959729$

Rochais, C., Lecoutey, C., Gaven, F., Giannoni, P., Hamidouche, K., Hedou, D., et al. (2015). Novel multitarget-directed ligands (MTDLs) with acetylcholinesterase (AChE) inhibitory and serotonergic subtype 4 receptor $\left(5-\mathrm{HT}_{4} \mathrm{R}\right)$ agonist activities as potential agents against Alzheimer's disease: the design of donecopride. J. Med. Chem. 58, 3172-3187. doi: 10.1021/acs. jmedchem.5b00115

Romero, A., and Marco-Contelles, J. (2017). Recent developments on multi-targetdirected tacrines for Alzheimer's disease: I. The pyranotacrines. Curr. Top. Med. Chem. 17, 3328-3335. doi: 10.2174/1568026618666180112155639 
Salloway, S., Sperling, R., Keren, R., Porsteinsson, A. P., van Dyck, C. H., Tariot, P. N., et al. (2011). A phase 2 randomized trial of ELND005, scylloinositol, in mild to moderate Alzheimer disease. Neurology 77, 1253-1262. doi: 10.1212/wnl.0b013e3182309fa5

Schneider, L. S., Mangialasche, F., Andreasen, N., Feldman, H., Giacobini, E., Jones, R., et al. (2014). Clinical trials and late-stage drug development for Alzheimer's disease: an appraisal from 1984 to 2014. J. Intern. Med. 275, 251-283. doi: 10.1111/joim.12191

Selkoe, D. J. (1991). The molecular pathology of Alzheimer's disease. Neuron 6, 487-498. doi: 10.1016/0896-6273(91)90052-2

Selkoe, D. J. (1997). Alzheimer's disease: genotypes, phenotypes and treatments. Science 275, 630-631. doi: 10.1126/science.275.5300.630

Selkoe, D. J., and Hardy, J. (2016). The amyloid hypothesis of Alzheimer's disease at 25 years. EMBO Mol. Med. 8, 595-608. doi: 10.15252/emmm.201606210

Shea, D., Hsu, C. C., Bi, T. M., Paranjapye, N., Childers, M. C., Cochran, J., et al. (2019). $\alpha$-Sheet secondary structure in amyloid $\beta$-peptide drives aggregation and toxicity in Alzheimer's disease. Proc. Natl. Acad. Sci. U S A 116, 8895-8900. doi: 10.1073 /pnas. 1820585116

Sigurdsson, E. M. (2016). Tau immunotherapy. Neurodegener. Dis. 16, 34-38. doi: 10.1159/000440842

Šimić, G., Babić Leko, M., Wray, S., Harrington, C., Delalle, I., JovanovMilošević, N., et al. (2016). Tau protein hyperphosphorylation and aggregation in Alzheimer's disease and other tauopathies and possible neuroprotective strategies. Biomolecules 6:6. doi: 10.3390/biom6010006

Stancu, I. C., Vasconcelos, B., Terwel, D., and Dewachter, I. (2014). Models of $\beta$-amyloid induced Tau-pathology: the long and "folded" road to understand the mechanism. Mol. Neurodegener. 9:51. doi: 10.1186/17501326-9-51

Stöhr, J., Condello, C., Watts, J. C., Bloch, L., Oehler, A., Nick, M., et al. (2014). Distinct synthetic A $\beta$ prion strains producing different amyloid deposits in bigenic mice. Proc. Natl. Acad. Sci. U S A 111, 10329-10334. doi: 10.1073/pnas. 1408968111

Suzuki, K., Iwata, A., and Iwatsubo, T. (2017). The past, present and future of disease-modifying therapies for Alzheimer's disease. Proc. Jpn. Acad. Ser. B Phys. Biol. Sci. 93, 757-771. doi: 10.2183/pjab.93.048

Teranishi, Y., Inoue, M., Yamamoto, N. G., Kihara, T., Wiehager, B., Ishikawa, T., et al. (2015). Proton myo-inositol cotransporter is a novel $\gamma$-secretase associated protein that regulates $A \beta$ production without affecting Notch cleavage. FEBS J. 282, 3438-3451. doi: 10.1111/febs.13353

Thapa, A., and Carroll, N. J. (2017). Dietary modulation of oxidative stress in Alzheimer's disease. Int. J. Mol. Sci. 18:E1583. doi: 10.3390/ijms18071583

Umar, T., and Hoda, N. (2017). Alzheimer's disease: a systemic review of substantial therapeutic targets and the leading multi-functional molecules. Curr. Top. Med. Chem. 17, 3370-3389. doi: 10.2174/ 1568026618666180112161024

Van der Schyf, C. J. (2011). The use of multi-target drugs in the treatment of neurodegenerative diseases. Expert Rev. Clin. Pharmacol. 4, 293-298. doi: $10.1586 /$ ecp. 11.13

van Dyck, C. H. (2018). Anti-amyloid- $\beta$ monoclonal antibodies for Alzheimer's disease: pitfalls and promise. Biol. Psychiatry 83, 311-319. doi: 10.1016/j. biopsych.2017.08.010

Viegas, C. Jr., Bolzani Vda, S., Barreiro, E. J., and Fraga, C. A. (2005). New anti-Alzheimer drugs from biodiversity: the role of the natural acetylcholinesterase inhibitors. Mini Rev. Med. Chem. 5, 915-926. doi: 10.2174/138955705774329546

Viegas-Junior, C., Danuello, A., da Silva Bolzani, V., Barreiro, E. J., and Fraga, C. A. (2007). Molecular hybridization: a useful tool in the design of new drug prototypes. Curr. Med. Chem. 14, 1829-1852. doi: 10.2174/092986707781058805

Walker, L. C., Ibegbu, C. C., Todd, C. W., Robinson, H. L., Jucker, M., LeVine, H. III., et al. (2005). Emerging prospects for the disease-modifying treatment of Alzheimer's disease. Biochem. Pharmacol. 69, 1001-1008. doi: $10.1016 /$ j.bcp.2004.12.015

Walter, J., Kemmerling, N., Wunderlich, P., and Glebov, K. (2017). $\gamma$-secretase in microglia-implications for neurodegeneration and neuroinflammation. J. Neurochem. 143, 445-454. doi: 10.1111/jnc.14224

Wang, J. Z., Xia, Y. Y., Grundke-Iqbal, I., and Iqbal, K. (2013). Abnormal hyperphosphorylation of tau: sites, regulation, and molecular mechanism of neurofibrillary degeneration. J. Alzheimers Dis. 33, S123-S139. doi: 10.3233/JAD-2012-129031

Wenzel, T. J., and Klegeris, A. (2018). Novel multi-target directed ligand-based strategies for reducing neuroinflammation in Alzheimer's disease. Life Sci. 207, 314-322. doi: 10.1016/j.lfs.2018.06.025

Wilkins, H. M., and Swerdlow, R. H. (2016). Relationships between mitochondria and neuroinflammation: implications for Alzheimer's disease. Curr. Top. Med. Chem. 16, 849-857. doi: 10.2174/1568026615666150827095102

Wisniewski, T., and Drummond, E. (2016). Developing therapeutic vaccines against Alzheimer's disease. Expert Rev. Vaccines 15, 401-415. doi: $10.1586 / 14760584.2016 .1121815$

Xia, W. (2019). $\gamma$-secretase and its modulators: twenty years and beyond. Neurosci. Lett. 701, 162-169. doi: 10.1016/j.neulet.2019.02.011

Zhang, Y., Li, P., Feng, J., and Wu, M. (2016). Dysfunction of NMDA receptors in Alzheimer's disease. Neurol. Sci. 37, 1039-1047. doi: 10.1007/s10072-0162546-5

Zhang, T., Loschwitz, J., Strodel, B., Nagel-Steger, L., and Willbold, D. (2019). Interference with amyloid- $\beta$ nucleation by transient ligand interaction. Molecules 24:E2129. doi: 10.3390/molecules24112129

Zhang, P., Xu, S., Zhu, Z., and Xu, J. (2019). Multi-target design strategies for the improved treatment of Alzheimer's disease. Eur. J. Med. Chem. 176, 228-247. doi: 10.1016/j.ejmech.2019.05.020

Conflict of Interest: GF and FT have an issued (0001383392) and a pending patent (102019000010722), both related to this work. MS has a pending patent (102019000010722) related to this work.

The remaining authors declare that the research was conducted in the absence of any commercial or financial relationships that could be construed as a potential conflict of interest.

Copyright (C) 2019 Catania, Giaccone, Salmona, Tagliavini and Di Fede. This is an open-access article distributed under the terms of the Creative Commons Attribution License (CC BY). The use, distribution or reproduction in other forums is permitted, provided the original author(s) and the copyright owner(s) are credited and that the original publication in this journal is cited, in accordance with accepted academic practice. No use, distribution or reproduction is permitted which does not comply with these terms. 